\title{
Functional Imaging of Decision Conflict
}

\author{
Jean-Baptiste Pochon, ${ }^{1 \star}$ Jason Riis, ${ }^{2 \star}$ Alan G. Sanfey, ${ }^{3 \star}$ Leigh E. Nystrom, ${ }^{4}$ and Jonathan D. Cohen ${ }^{4}$ \\ ${ }^{1}$ INSERM Avenir IFR70 and U610, Hôpital Pitié-Salpêtrière, F-75103 Paris, France, ${ }^{2}$ Harvard Business School, Boston, Massachusetts $02163,{ }^{3}$ Department \\ of Psychology, University of Arizona, Tucson, Arizona 85721, and ${ }^{4}$ Center for the Study of Brain, Mind and Behavior, Princeton University, Princeton, New \\ Jersey 08544
}

Decision conflict occurs when people feel uncertain as to which option to choose from a set of similarly attractive (or unattractive) options, with many studies demonstrating that this conflict can lead to suboptimal decision making. In this article, we investigate the neurobiological underpinnings of decision conflict, in particular, the involvement of the anterior cingulate cortex (ACC). Previous studies have implicated the ACC in conflict monitoring during perceptual tasks, but there is considerable controversy as to whether the ACC actually indexes conflict related to choice, or merely conflict related to selection of competing motor responses. In a functional magnetic resonance imaging study, we dissociate the decision and response phases of a decision task, and show that the ACC does indeed index conflict at the decision stage. Furthermore, we show that it does so for a complex decision task, one that requires the integration of beliefs and preferences and not just perceptual judgments.

Key words: conflict; decision; fMRI; anterior cingulate cortex; preferences; attractiveness

\section{Introduction}

When choosing between alternatives of similar overall desirability, decision makers may find themselves unsure about which option is preferred, an experience of indecision that has been termed "decision conflict." There is considerable evidence that decision conflict can lead to suboptimal choices. For example, Dhar (1997) has shown that people are more likely to choose the status quo over two equally attractive options, even if both of these options are individually preferred to the status quo. Similarly, Nowlis et al. (2002) demonstrated that decision conflict leads to choosing risk-minimizing options at the expense of maximizing utility. In the present article, we investigate the neurobiological underpinnings of decision conflict, particularly the involvement of the anterior cingulate cortex (ACC).

The involvement of the ACC in monitoring conflict has been hypothesized in a theory of the adaptive regulation of cognitive control put forward by Botvinick et al. (2001). Broadly speaking, this theory proposes that the ACC is engaged by the detection of simultaneous activation of mutually competing alternatives during response generation. On detection of conflict, the ACC signals other brain regions involved in the execution of control to resolve the conflict. Most experimental tests of this hypothesis have used relatively low-level, perceptual decision-making tasks, such as deciding the direction of a target stimulus (Eriksen flanker task), or simple recognition and retrieval tasks, such as

Received Sept. 13, 2007; revised Jan. 7, 2008; accepted Feb. 1, 2008

This work was supported by National Institutes of Mental Health Grant P50MH062196, Fondation Fyssen, and the Center for Health and Wellbeing at Princeton University. We thank Cara Buck, Jennifer Edwards, and Peter Hu for valuable research assistance.

*J.-B.P., J.R., and A.G.S. contributed equally to this work.

Correspondence should be addressed to Dr. Alan G. Sanfey, Department of Psychology, University of Arizona, 1503 East University Boulevard, Building 68, Tucson, AZ 85721. E-mail: asanfey@u.arizona.edu.

D01:10.1523/JNEUROSCI.4195-07.2008

Copyright $\odot 2008$ Society for Neuroscience $\quad$ 0270-6474/08/283468-06\$15.00/0 word stem completion (Palmer et al., 2001) and verb generation (Thompson-Schill et al., 1997; Barch et al., 2000).

Here, we address two open questions concerning the role of ACC in conflict monitoring. First, we examine whether the proposed conflict monitoring role extends to more complex decisions that involve the integration of higher-level beliefs and preferences. Specifically, does the ACC detect the "decision conflict" that occurs when two options are similar in desirability? We addressed this by measuring ACC activity while male participants made choices between female faces, with the task being to choose which face in a given pair would be considered more attractive by other males. Extensive pretesting allowed us to reliably predict which pairs would be associated with high and low conflict, respectively, and we predicted more ACC activity during highconflict trials than low-conflict trials.

Second, we asked whether conflict detection is related to activity at the decision stage itself. Several researchers have suggested that the ACC plays a role in motor response selection (Milham et al., 2001; van Veen et al., 2001; Zysset et al., 2006), and because decision conflict may engender simultaneous preparation of competing motor responses, ACC activity could reflect conflict among incompatible motor responses rather than decision conflict per se. We addressed this question by temporally separating the decision and motor response phases of the task and by making the association between a given choice and the corresponding response highly unpredictable, thus discouraging motor preparation. Additionally, on some trials no motor response was required. These manipulations allowed us to dissociate the decision and response phase of the trials.

\section{Materials and Methods}

Stimuli and task design. Images of female faces were used as stimuli (eight-bit grayscale; 242 width $\times 310$ height). Attractiveness ratings of the faces were obtained in a pretest of 12 male participants who rated the faces on a nine-point scale from very unattractive (1) to very attractive 
(9). Faces were then paired and the pairs were rated by another group of 17 male participants on a different nine-point scale, ranging from very dissimilar in attractiveness (1) to very similar in attractiveness (9). Using these sets of ratings, 120 pairs were constructed to either consist of two similarly attractive faces ("high-conflict-attractive"), two similarly unattractive faces ("high-conflict-unattractive"), or one attractive face and one unattractive face ("low conflict"). In the scanner, the 120 pairs were presented in five runs of 24 trials, with short breaks between runs. On each trial, the participant's task was to choose which of the two faces a majority of other undergraduate males would find most attractive (a pretest revealed that this task would induce more experienced conflict than simply deciding on one's own preference).

To allow us to assess the involvement of decision conflict independent of response conflict, the decision and response phases of each trial were separated. In the decision phase, the pair of faces was presented for several seconds and the participant was supposed to mentally make his choice during this time. The duration of this decision phase, or pair presentation durations, was randomly jittered to be either 3.6, 5.4, or $7.2 \mathrm{~s}$ (a negative exponential distribution was used to determine the duration, so the longer duration occurred for only $\sim 5 \%$ of the trials, with the remainder being roughly evenly split between the short and medium durations.) After this decision phase, a randomly selected letter appeared beneath each face. When the letters appeared, the participant was to vocalize the letter associated with the face he had chosen. The participant was instructed to minimize jaw movement during vocalization. On 50\% of trials, response letters did not appear, so a response was not required. The participant had no way of knowing on which trials a response would be required, and on trials when the response letters did appear, the participant had no way of knowing in advance what the response letters would be. The purpose of this design was to eliminate response conflict during the decision phase of the task. Each trial lasted between 10.8 and $18 \mathrm{~s}$ and the total duration of a run was $408.6 \mathrm{~s}$.

An infrared eye-tracking system (Applied Science Laboratories, Bedford, MA) was used to record eye movements while participants performed the task. Analyses of the eye-tracking data were performed using a Matlab 7 script developed by the authors. All of the stimuli were presented with E-prime (Psychology Software Tools, Pittsburgh, PA) on a personal computer and back-projected onto a screen using a liquid crystal display projector and viewed by participants through a mirror mounted on glasses. Participants' verbal responses were recorded in the scanner using a magnetic resonance imaging (MRI) scanner-safe microphone. Verbal responses were digitally recorded and noise-filtered using Audacity software (http://audacity.sourceforge.net/). As a measure of response time, the temporal latency of the vocalizations (i.e., time from the appearance of the letters on the screen until onset of vocalization) were recorded for each trial.

After each scanning session, participants were shown the pairs of faces two more times. First, they were asked to rate the similarity of attractiveness of the two faces in each pair, on the same nine-point scale used in the pretest. When the pairs were presented a second time, the participants were asked to rate the level of attractiveness of each face in the pair, again using the scale described earlier. Participants were then debriefed and dismissed.

fMRI data acquisition. Imaging was performed in the Psychology Department at Princeton University with a 3-Tesla head-dedicated MRI system (Magnetom Allegra; Siemens, Erlangen, Germany). Functional MRI (fMRI) images were acquired using an echo-planar imaging pulse T2*-weighted sequence [field of view (FOV), $192 \times 192 \mathrm{~mm} ; 64 \times 64$ matrix; repetition time (TR), $1800 \mathrm{~ms}$; echo time (TE), $30 \mathrm{~ms}$; flip angle, $80^{\circ}$. Twenty-eight contiguous axial slices were acquired (thickness of 4 $\mathrm{mm}$ without gap; in-plane resolution, $3 \times 3 \mathrm{~mm}$ ). The brain was covered from the vertex to the middle of the cerebellum. Structural MRI images were obtained using an magnetization-prepared rapid gradient echo T1weighted sequence (FOV, $256 \times 256 \mathrm{~mm} ; 256 \times 256$ matrix; inversion recovery technique, $1100 \mathrm{~ms}$; TE, $4.4 \mathrm{~ms}$; TR, $2500 \mathrm{~ms}$; flip angle, $8^{\circ}$; 176 sagittal slices).

fMRI data analysis. All fMRI analyses were performed using SPM2 (Wellcome Department, London, UK) running on a Matlab 7 platform (MathWorks, Natick, MA). Images were successively corrected for mo- tion and slice time acquisition, and normalized to the Montreal Neurological Institute (MNI) template using a 12-parameter linear transformation defined from the normalization of the anatomical scan to the MNI T1 template. Images were then smoothed using a Gaussian filter of 10 $\mathrm{mm}$ full-width at half maximum. Motion parameters were stored and used as nuisance variables in the generalized linear model (GLM) analysis. Additional nuisance variables were used to assess the effects of eye movements, such that each time a saccade was detected, a standard hemodynamic response was added. Assuming the linearity of the hemodynamic response, this nuisance covariate accounts for the variance of the signal linked with the saccadic activity.

For each experiment, statistical parametric maps (SPMs) were computed using the general linear model, with separate hemodynamic response functions for each task condition and for each phase (decision and response). Functions that model the same level of conflict (high, low) and attractiveness (high, low) were pooled together for the GLM analysis. In the parametric analysis, all events of the decision phase were pooled together, but the amplitude of each event varied depending on the rated similarity (a proxy for conflict) of the pairs taken after scanning.

For each analysis, an SPM $\{F\}$ map was obtained, reflecting significant activated voxels according to the model used ( $p<0.001$ ). To test hypotheses about regionally specific condition effects, the estimates were compared using linear contrasts. The resulting set of voxel values for each contrast constituted an SPM $\{\mathrm{T}\}$ map. Random effect analysis was then performed using $t$ test analysis across the participants on the contrasts images of interest. The resulting set of $t$ values had thresholds of $p<$ 0.001 with a spatial extent threshold of 20 contiguous voxels. fMRI results presented will mainly be focused on the no-response trials (50\%), excluding confounding factors such as blood oxygen level-dependent (BOLD) motor-related activity and the possible interfering magnetic susceptibility modification caused by jaw movements.

\section{Results}

\section{Postscanner check of the conflict manipulation}

In the postscanner behavioral task, participants were shown the pairs of faces again and asked to rate, for each pair, how similar the two faces were with respect to their level of attractiveness. These ratings were very consistent with our preclassification of the pairs as high conflict or low conflict (see Materials and Methods). Pictures in pairs that were preclassified as high conflict were rated as very similar in attractiveness (mean, 7.1; SE, 0.5) on a nine-point "similarity of attractiveness" scale. Pairs that were preclassified as low conflict had much lower ratings $\left(t_{(15)}>22\right.$; $p<10^{-12}$; mean 2.3; SE, 0.3), suggesting that, as intended, one picture in each pair was much more attractive than the other.

Choices made in the scanner were highly consistent with those made outside the scanner. The choices made outside the scanner were only made implicitly: participants did not indicate which face was more attractive, but they did rate the attractiveness of each face in the pair on a nine-point scale. Across participants, on trials where the ratings of the two faces differed by just one point, the face with the higher rating had been chosen as more attractive in the scanner on $91 \%$ of these trials. When the ratings differed by two points, consistency with the scanner response was $95 \%$. And when the ratings differed by three or more points, the consistency was at least $96 \%$. This suggests that the choices made in the scanner reflected systematic judgments.

\section{Vocalization latency}

Across participants, the mean vocalization latencies did not differ significantly between the high-conflict-attractive pairs (mean, $699 \mathrm{~ms}$; SE, $175 \mathrm{~ms}$ ), the high-conflict-unattractive pairs (mean, $769 \mathrm{~ms}$; SE, $181 \mathrm{~ms}$ ), or the low-conflict pairs (mean, $656 \mathrm{~ms}$; $\mathrm{SE}, 145 \mathrm{~ms}$ ) (all paired $t$ tests, $<2.0$, not significant). 


\section{ACC activation}

Across both response and no-response trials in the scanner, there was a large ACC activation when comparing the highconflict to the low-conflict trials (collapsing across the high- and low-attractive pairs). As shown in Figure $1 a$, this activity was centered on Talairach coordinates $[x=-3 ; y=20 ; z=43$; Brodmann's area (BA) 32]. This cluster of activation was mainly circumscribed within the dorsal aspect of ACC. However, it also extended to the ventral aspect of the medial frontal gyrus, within the presupplementary motor area (BA 6). When considering only the no-response trials the same cluster within the ACC was found (Fig. 1b, Table 1). In an additional analysis, using each participant's postscanner evaluation of the pairs as a parametrically varying covariate, the same ACC area was activated. In these last two analyses, activation also extended to the anteroventral aspect of the dorsal ACC (Fig. 1b,c, Table 1).

The time course of ACC activation in high- and lowconflict trials is illustrated in Figure $2 a$. These time course curves reveal that hemodynamic response within ACC increased immediately after picture pairs were presented to participants. Responses for high-conflict trials (red curves) were almost twice as strong as the responses observed for the lowconflict trials (black curves). The duration of the decision phase varied randomly between trials (with possible durations of $3.6,5.4$, or $7.2 \mathrm{~s} ; 2,3$, or 4 TRs), so participants could not easily anticipate when a response would be required. Figure $2 a$ also reveals that trials with the shortest decision phase duration (3.6 s; dashed lines), showed the same pattern as the full set of trials (i.e., collapsing across trials with a 3.6, 5.4, and $7.2 \mathrm{~s}$ decision phase duration; solid lines). Because these shorter trials would have included the greatest proportion of "predecision" time, it is encouraging that the differential pattern of ACC activation is observed even in these trials where postdecision processes are less likely to have been involved.

Additional evidence for increased ACC activity during the decision phase is seen in Figure $2 b$, which shows the time course curves of ACC activation (orange lines) and of facial motor area activation (purple lines). The solid lines, which plot activation for trials when a response was required, reveal that ACC activation began almost $3.6 \mathrm{~s}$ before facial motor area activation, suggesting that ACC activation was indexing decision processes that occurred well before the participants vocalized their responses. It is of course possible the ACC activation is indexing conflict in motor preparation, and that additional ACC activation on highconflict trials is capturing additional motor preparation. But this seems unlikely because it is unclear just what kind of motor preparation could be occurring when the participants did not know what kind of motor response would be required (i.e., which letter vocalization was associated with the different faces), nor did they even know if any motor response would be required.

Interestingly, Figure $2 b$ also shows that there was more ACC activity on response trials than on no-response trials, but primarily at time points at which (and after which) the response was actually being made (for TRs 4, 5, and 6: $t=4.96, p<0.001 ; t=$ $6.84, p<0.001$; and $t=4.66, p<0.001$, respectively) This activation is in the same peak area as was observed in the high- versus low-conflict analysis. There was also an unexpected activation difference on TR $1(t=2.61, p<0.05)$, but there was no differ- ence on TR 2 or TR 3 ( $t=1.34$ and $t=0.33$, respectively). Because no-response and response trials were indistinguishable to participants at TR1, we suspect that this difference results from random variation. The ACC difference in the late TRs suggests that the ACC may play some additional role in the vocalization of responses. However, this role appears to be independent of its role in decision conflict, because ACC activation differences between high- and low-conflict trials were still seen for no-response trials only (Figs. 1b, 2a).

\section{Activation outside the ACC}

In addition to activation within the ACC, all three contrasts (all trials, only no response trials and parametric analysis) reveal activity within the right orbitofrontal cortex (BA 11/47), the right inferior frontal gyrus (BA 9 or 45/46), the striate visual cortex (BA 17/18), and within fusiform gyri (BA 37). For the parametric analysis, minor differences were also observed in the left parahippocampal gyrus (BA 35) and in the left anterior insular cortex (BA 44/47) (for details of all active areas, see Table 1).

\section{Eye movements}

Given the high visual demands of the task, and the proximity of the ACC to the supplementary eye fields, it seems possible that the difference in ACC activation between high- and low-conflict trials may be indexing differences in eye movements rather than differences in decision conflict per se. The inclusion of eye movements as nuisance variables in the GLM, however, argues against this possibility. ACC activation differences between high- and low-conflict trials are observed despite the inclusion of such nuisance variables.

A direct analysis of eye movements also fails to support the suggestion that they are involved in ACC activation. Saccadic movement counts were extracted from the time course positions of the participants' glances. For the trials with the shorter decision phase (3.6 s), there were not significantly more saccadic movements on the high-conflict than low-conflict trials (mean, 5.2 and 4.9, respectively; $p>0.12$ ), even though there was increased ACC activation on the high-conflict trials of this duration (Fig. 2a). For trials with longer decision phase duration (5.4 and $7.2 \mathrm{~s})$, there were more eye movements on high-conflict trials (mean, 6.5 vs $5.4 ; p<0.001$ ), but these eye movements were not associated with the large differences in ACC activation that were also observed. This is seen in a factorial mixed-model ANOVA (duration by conflict by saccades, with repeated measures) for the ACC signal averaged over the decision-making period for the no-response trials. We found a significant effect of duration $(p<$ 0.011 ) and conflict $(p<0.001)$. However, no significant effect was observed for the saccades count $(p=0.321)$.

Additional evidence that eye movements were not the cause of 
Table 1. Activation during the no-response trials

\begin{tabular}{|c|c|c|c|c|c|c|c|}
\hline \multirow[t]{2}{*}{ Location } & \multirow[t]{2}{*}{ Laterality } & \multirow[t]{2}{*}{ Brodmann's areas } & \multicolumn{3}{|c|}{ Talairach's coordinates } & \multirow[t]{2}{*}{$t$ values } & \multirow[t]{2}{*}{ Cluster size (voxels) } \\
\hline & & & $x$ & $y$ & $z$ & & \\
\hline \multicolumn{8}{|l|}{ High- versus low-conflict contrasts } \\
\hline \multicolumn{8}{|l|}{ Prefrontal cortex } \\
\hline Anterior cingulate cortex & R and $L$ & BA $6 / 32$ & -3 & 20 & 43 & 4.49 & 253 \\
\hline Medial frontal cortex & R and $L$ & BA $9 / 32$ & -6 & 39 & 23 & 3.61 & 253 \\
\hline Lateral prefrontal cortex & $\mathrm{R}$ & BA 9 & 42 & 4 & 27 & 6.22 & 71 \\
\hline Orbito frontal cortex & $\mathrm{R}$ & BA 11/47 & 39 & 32 & 4 & 5.27 & 179 \\
\hline \multicolumn{8}{|l|}{ 0thers } \\
\hline Fusiform gyrus & $\mathrm{R}$ & BA 37 & 42 & -61 & 7 & 4.63 & 61 \\
\hline Striate visual cortex & $\mathrm{L}$ & BA 17/18 & -9 & -79 & 1 & 4.73 & 57 \\
\hline \multicolumn{8}{|l|}{ Conflict parametric analysis } \\
\hline \multicolumn{8}{|l|}{ Prefrontal cortex } \\
\hline Anterior cingulate cortex & R and $L$ & BA 32 & 3 & 20 & 49 & 4.47 & 242 \\
\hline Anterior cingulate cortex & R and $L$ & BA 32 & 6 & 29 & 1 & 4.78 & 94 \\
\hline Medial frontal cortex & R and $L$ & BA $9 / 32$ & 9 & 36 & 26 & 4.03 & 242 \\
\hline Lateral prefrontal cortex & $\mathrm{R}$ & BA 9/46 & 48 & 22 & 24 & 5.22 & 341 \\
\hline Orbito frontal cortex & $\mathrm{R}$ & BA 11/47 & 30 & 40 & -12 & 4.98 & 103 \\
\hline Anterior insular cortex & $\mathrm{L}$ & BA 44/47 & -39 & 23 & 2 & 4.90 & 163 \\
\hline \multicolumn{8}{|l|}{ 0thers } \\
\hline Fusiform gyrus & $\mathrm{R}$ & BA 37 & 45 & -64 & 7 & 5.11 & 143 \\
\hline Fusiform gyrus & $\mathrm{L}$ & BA 37 & -50 & -64 & 6 & 5.25 & 161 \\
\hline Striate visual cortex & $\mathrm{R}$ & BA 17/18 & 18 & -79 & 1 & 5.36 & 93 \\
\hline Parahippocampal gyrus & $\mathrm{L}$ & BA 35 & 27 & -24 & -14 & 6.51 & 88 \\
\hline
\end{tabular}

Areas showing greater activation for high-conflict trials than low-conflict trials, on trials where no response was required. The top half of the table describes activity as a result of the direct contrast of high versus low conflict, whereas the bottom half describes areas active when examining conflict parametrically based on participants responses. $t$ value thresholds of $p<0.001$ with a spatial extent threshold of 20 contiguous voxels.

the increased ACC activity in high-conflict trials comes from the fact that there was no evidence of increased activation in the frontal eye fields (FEFs), as the associated voxels did not appear even at a very lenient threshold $(p<0.1)$. The signal recorded within the FEF is shown in Figure $2 d$ and did not reveal any differences between conditions.

\section{Attractive pairs versus unattractive pairs}

When comparing the two categories of high-conflict trials (that is, those with two attractive faces and those with two unattractive faces), differences were observed in activation in the most dorsal aspect of the ACC (BA 6) cluster described above. Attractive faces led to a higher activation of the ACC compared with unattractive faces (Fig. 2e). Consistent with this finding, an additional analysis of behavioral data revealed that "similarity of attractiveness" was rated as significantly higher for attractive pairs than for unattractive pairs $(p<0.001)$, suggesting that decisions about the attractive pairs may in fact have been more difficult, and induced more conflict, than decisions about unattractive pairs. It is not clear if this is because of the specific stimuli we chose, or if it is a more general finding about these kinds of choices. The fusiform gyri (BA 19/37) and the caudate nuclei also showed more activation for attractive pairs. Conversely, extensive activation within the posterior and middle insular cortex, the middle temporal gyri (BA 21/22), right anterior inferior insula, and within both postcentral gyri (BA 1/2/3) was observed when comparing unattractive pairs to attractive ones.

\section{Discussion}

In an engaging decision task, we found greater ACC activation when participants faced difficult (high conflict) decisions than when they faced easier (low conflict) decisions. Because we dissociated the decision and response phases of the task, the results strongly suggest that these ACC differences were caused by actual decision conflict, and not response conflict.

Several other studies have demonstrated ACC activation associated with decision making in higher level cognitive tasks. For example, in a study of economic social interactions by Sanfey et al. (2003), participants had to respond to a monetary offer made by a partner, and there was greater ACC activation when the offer caused increased conflict between accept and reject responses. Similarly, Greene et al. (2004) found increased ACC activation when the participants made more difficult moral decisions. Our results are also consistent with previous work by De Martino et al. (2006), who found ACC activation corresponding to decisions that were incongruent with an experimentally provided frame.

Studies such as these, however, were not specifically designed to test the role of the ACC. Therefore, the results of these studies cannot eliminate the possibility that the ACC activity observed was the result of conflict between competing motor responses representing the different decisional outcomes, rather than decisional conflict as such. Indeed, some authors directly suggest that ACC activity reflects motor preparation, independent of conflict. For example, a recent study (Zysset et al., 2006) suggests that ACC activity reflects motor preparation before the participant making an overt response. However, this study did not include trials with a high degree of decision conflict; that is, trials in which there is no dominating option. Thus, although this study suggests that the ACC may be responsive to motor preparation, it does not provide evidence against the hypothesis that the ACC is also responsive to decision conflict. In the present study we dissociated decision from response processes, and observed ACC activity associated with high-conflict trials in during the decision phase. Accordingly we can conclude not only that the ACC is more active during high-conflict decisions, but also that this activation is related to decision processes and not merely to motor response preparation processes or motor conflict.

A number of other studies have suggested that the ACC is involved in different aspects of decision behavior, for example risk versus ambiguity processing (Cohen et al., 2005; Krain et al., 2006; Paulus and Frank, 2006), reward processing (Kennerley et al., 2006), or action selection (Rushworth et al., 2007). The findings of those studies are largely orthogonal to the ones here and 
future investigations should examine different factors in the same study. A design similar to the current one, for example, could manipulate decision difficulty in different ways, both in terms of option attractiveness and in terms of option likelihood. This would allow a simultaneous mapping of different decision processes: risk processing, reward processing, and conflict processing.

We also observed activation outside ACC when comparing high- versus lowconflict trial types. In particular, right inferior and lateral frontal gyri, anterior insula, orbitofrontal cortex and striate visual cortex all showed increased activity for more difficult choices. Several of these areas have shown ACC coactivation in other studies (Botvinick et al., 2001; Greene et al., 2004). Speculatively, these activations could reflect the recruitment of additional resources and effort in the higher conflict trials, though further research will be needed to more fully understand the involvement of these areas.

Computational models have implemented conflict in terms of the energy of representation (Botvinick et al., 2001) in which case two highly activated but competing representations are associated with greater conflict than two competing representations that are less highly activated. Thus, our finding that decisions between attractive faces were associated with greater ACC activity than those involving unattractive faces could reflect greater decisionrelated activity in response to attractive versus unattractive faces. But again, this issue requires additional research.

The results of the present study have an important role to play in attempting to localize aspects of decision making to specific neural structures and processes. The results also have the potential to lead to useful behavioral insights. For example, Tom et al. (2007) found that individual differences in loss aversion were predicted by differential activation in the ventral medial prefrontal cortex and in the striatum. It would be interesting if individual differences on psychological constructs such as indecisiveness and ambiguity aversion (Fox and Tversky, 1995) could similarly be predicted by activation differences in the ACC. Additionally, in future work, we hope to examine the role of ACC activity in actual decision behaviors such as status quo bias and risk aversion. Both are thought to be driven by decision conflict and both are non-normative. Are such non-normative choices associated with affective processing (cf. Greene et al., 2004), or are they associated with minimal recruitment of higher cognitive processes? Imaging studies that manipulate the conflict in decision tasks and then examine the relationship between choice behavior and activation would be able to address such questions.

\section{Time-course curves of BOLD responses}

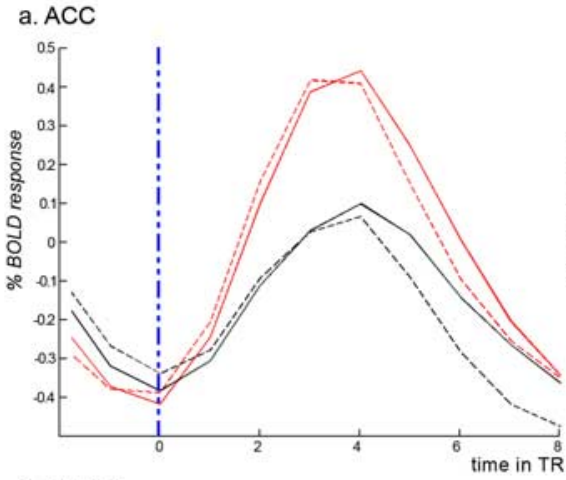

b. Primary motor cortex and ACC

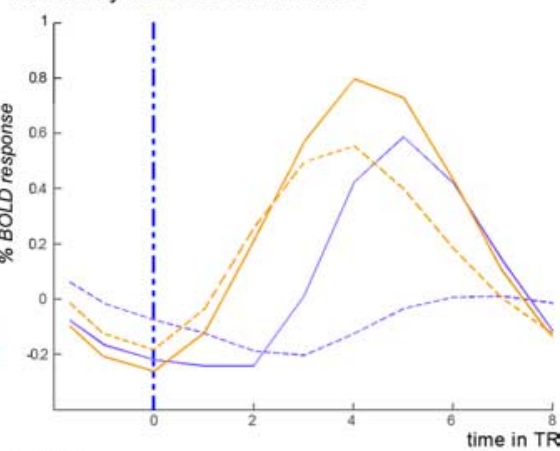

d. FEF
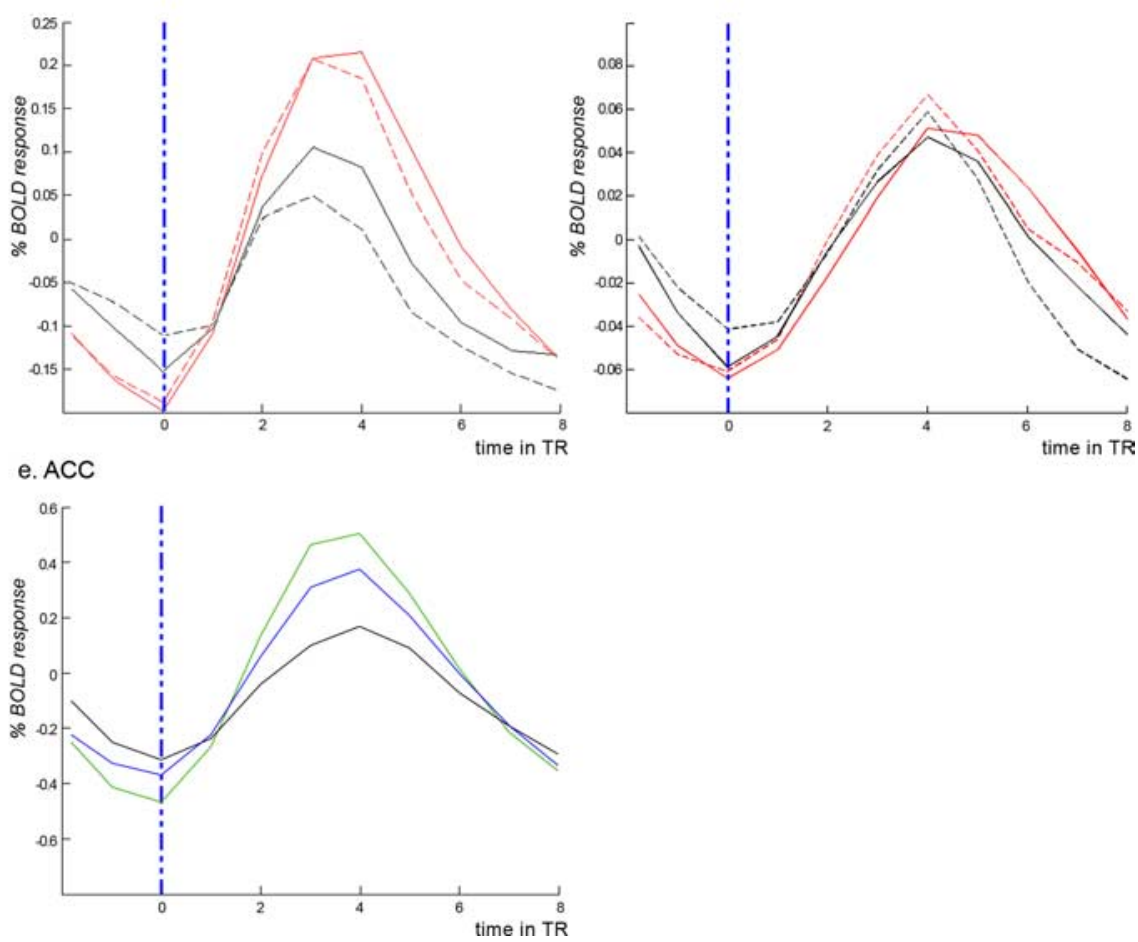

Figure 2. Averaged and fitted time course curves of $f M R I$ signal (BOLD response). In all panels, the vertical blue dashed line corresponds to the appearances of the faces. TR, $1.8 \mathrm{~s}$. A, Signal within the ACC for the no response trials (red, high conflict; black, low conflict). Solid lines show activation for all trials combined, with dashed lines showing activation only for trials with the shortest decision phase duration (3.6s). B, Signal within the ACC (orange) and the primary motor cortex face area (purple); solid lines correspond to trials where a verbal motor response is required and dashed lines to trials without an overt response. C, Signal within dorsolateral prefrontal cortex (DLPFC) for the no response trials (red, high conflict; black, low conflict. Solid lines show activation for all trials combined, with dashed lines showing activation only for trials with the shortest decision phase duration (3.6s). D, Signal within FEF for the no response trials (red, high conflict; black, low conflict). Solid lines show activation for all trials combined, with dashed lines showing activation only for trials with the shortest decision phase duration ( $3.6 \mathrm{~s})$. $\boldsymbol{E}$, Signal within the ACC for the no response trials [green, high conflict (attractive); blue, high conflict (unattractive); black, low conflict].

\section{References}

Barch DM, Braver TM, Sabb FW, Noll DC (2000) Anterior cingulate and the monitoring of response conflict: Evidence from an fMRI study of overt verb generation. J Cogn Neurosci 12:298-309.

Botvinick MM, Braver TS, Carter CS, Barch DM, Cohen JD (2001) Conflict monitoring and cognitive control. Psychol Rev 108:624-652.

Cohen MX, Heller AS, Ranganath C (2005) Functional connectivity with anterior cingulate and orbitofrontal cortices during decision-making. Cognitive Brain Res 2 3:61-70.

De Martino B, Kumaran D, Seymour B, Dolan RJ (2006) Frames, biases, and rational decision-making in the human brain. Science 313:684-687.

Dhar R (1997) Consumer preference for a no-choice option. J Consumer Res 24:215-231. 
Fox CR, Tversky A (1995) Ambiguity aversion and comparative ignorance. Quart J Econom 110:585-603.

Greene JD, Nystrom LE, Engell AD, Darley JM, Cohen JD (2004) The neural bases of cognitive conflict and control in moral judgment. Neuron 44:389-400.

Kennerley SW, Walton ME, Behrens TEJ, Buckley MJ, Rushworth MFS (2006) Optimal decision making and the anterior cingulate cortex. Nat Neurosci 9:940-947.

Krain AL, Wilson AM, Arbuckle R, Castellanos FX, Milham MP (2006) Distinct neural mechanisms of risk and ambiguity: A meta-analysis of decision-making. NeuroImage 32:477-484.

Milham MP, Banich MT, Webb A, Barad V, Cohen NJ, Wszalek T, and Kramer AF. (2001) The relative involvement of anterior cingulate and prefrontal cortex in attentional control depends on nature of conflict. Brain Res Cogn Brain Res 12:467-473.

Nowlis SM, Khan BE, Dhar R (2002) Coping with ambivalence: The effect of removing a neutral option on consumer attitude and preference judgments. J Consumer Res 29:319-334.

Palmer ED, Rosen HJ, Ojemann JG, Buckner RL, Kelley WM, Petersen SE (2001) An event-related fMRI study of overt and covert word stem completion. NeuroImage 14:182-193.
Paulus MP, Frank LR (2006) Anterior cingulate activity modulates nonlinear decision weight function of uncertain prospects. NeuroImage 30:668-677.

Rushworth MF, Behrens TE, Rudebeck PH, Walton ME (2007) Contrasting roles for cingulate and orbitofrontal cortex in decisions and social behaviour. Trends Cogn Sci 11:168-176.

Sanfey AG, Rilling JK, Aronson JA, Nystrom LE, Cohen JD (2003) The neural basis of economic decision making in the Ultimatum Game. Science 300:1755-1758.

Thompson-Schill SL, D’Esposito M, Aguirre GK, Farah MJ (1997) Role of left inferior prefrontal cortex in retrieval of semantic knowledge: A reevaluation. Proc Natl Acad Sci USA 94:14792-14797.

Tom SM, Fox CR, Trepel C, Poldrack RA (2007) The neural basis of loss aversion in decision-making under risk. Science 315:515-518.

van Veen V, Cohen JD, Botvinick MM, Stenger VA, Carter CS (2001) Anterior cingulate cortex, conflict monitoring, and levels of processing. NeuroImage 14:1302-1308.

Zysset S, Wendt CS, Volz KG, Neumann J, Huber O, von Cramon DY (2006) The neural implementation of multi-attribute decision making: A parametric fMRI study with human subjects. NeuroImage 31: $1380-1388$. 